\title{
Time Synchronized High Performing Cluster-Based Routing Algorithm for Wireless Sensor Networks
}

\author{
*Huifang DENG, Tingting WANG \\ South China University of Technology, China \\ *hdeng2008@gmail.com
}

\begin{abstract}
In this paper, we investigated the data fusion routing algorithm and the time synchronization algorithm for the wireless sensor networks. First, we proposed a new data fusion routing algorithm - Low Energy Consumption Cluster-Based Routing Algorithm (LECCBRA). Simulation results show that the total energy can be effectively saved, and the balance in energy consumption between clusters can be achieved with LECCBRA, thus the network lifetime is extended. Second, we proposed a time synchronization algorithm - Clustering-based Time Synchronization (CBTS), which is suitable for LECCBRA. Performance analysis and simulation results show that the total energy can be effectively saved, and the accuracy can be improved.
\end{abstract}

Keywords: Data fusion routing algorithm; wireless sensor networks; low energy consumption cluster-based routing algorithm; clustering-based time synchronization; NS2

\section{Introduction}

As the basis for and key technique of Internet of Things (Commission of the European Communities, 2009; Kranenburg, 2007), wireless sensor network technology has gained more and more attention. Wireless Sensor Networks (WSN) (Ren et al., 2003) is a highly discipline-crossed, knowledge-integrated, widely attention-received research area. It involves sensor technology, communication technology, and embedded computing technology. It can be widely used in areas such as environmental protection, industrial production, disaster relief, medical treatment, military security, and space exploration etc. In the Wireless Sensor Networks, resources on each node are very limited in terms of battery power, processing capability, storage capacity, and communication bandwidth. Thus to improve energy-use efficiency has become the focus of the research for sensor network. Data fusion is an important way to save energy in Wireless Sensor Networks. Both LEACH (Heinzelman et al., 2000) and LEACH-C (Siva \& Ma, 2005) are data fusion methods based on hierarchical philosophy. They use clustering method to highlight the importance of data fusion. In addition, the clock synchronization is the basis for data processing in WSN, and different data fusion between nodes' time demands different system synchronization accuracy. According to the limited bandwidth and limited computational resources in WSN, we must design an effective clock synchronization and data fusion algorithm to ensure each node having the data synchronization and not losing the authenticity. In this paper, we investigate the data fusion routing algorithm and the time synchronization algorithm for the wireless sensor networks. First, we proposed a new data fusion routing algorithm - Low Energy Consumption Cluster-Based Routing Algorithm (LECCBRA). This algorithm (protocol) offers improvements in selection of next cluster head, re-partition of network, and inter-cluster routing. Second, we proposed a time synchronization algorithm - Clustering-based Time Synchronization (CBTS), which is suitable for LECCBRA.

\section{LECCBRA Protocol}

The Basic Concept of LECCBRA: Considering the imperfection of LEACH protocol and taking the advantages of LEACH-C protocol, LECCBRA is proposed to improve cluster head selection and inter-cluster routing. The basic concept is as follows:

a) Cluster head selection: Only for the first round, the network is clustered by the algorithm of LEACH-C protocol so as to generate clusters. Based on this, the entire network is split into uniform regions, and it retains the partition in the next $\mathrm{N}$ rounds. In each of $\mathrm{N}$ rounds, when data transmission is done, the current cluster head decides the next cluster head by considering the remaining energies and locations of cluster members. A member will become the next cluster head if it has more energy 
remaining and is closer to the center of mass. And the current cluster head will broadcast this message to all members. At the end of the round $\mathrm{N}$, each cluster head collects the cluster energy and positional information of the remaining members and transmits, directly or indirectly to the base station.

b) Inter-cluster routing: The cluster head communicates with the base station by multi-hop mode. The base station finds the shortest routing between the base station and the rest of cluster heads, using a global shortest path algorithm named Floyd-warshall (Floyd, 1962). Then it broadcasts the routing.

\section{Description of LECCBRA}

c) Cluster head selection: The cluster head selection algorithm of LECCBRA protocol is shown as follows:

(1) If $r==1$, then all nodes send the information of their surplus energies and locations directly to the base station.

(2) If $r \bmod (N+1)==1$, then the base station generates the set of cluster heads and the positional information of the clusters, according to LEACH-C protocol. Then the base station broadcasts the first set of cluster heads and the positional information. Then the phase of data transmission follows.

(3) Consider the case of $r$ mod $(N+1) !=1$. In the last round, the cluster head has specified the node of the next cluster head, so in this round, all the cluster members send the message to the corresponding cluster head, informing the head that they have become its member. After the cluster head receives the messages from all members, the data transmission phase is done.

(4) After the completion of the data transmission, if $r \bmod (N+1) !=0$, all cluster members send their surplus energies to their own cluster head. The cluster head chooses the member having more remaining energy and being closer to the center of mass as the next cluster head. The method for calculating the composite value of the next cluster head is as follows:

$C_{i}=c_{1} E_{i}+c_{2} D_{i}$

$$
D i=\frac{d_{N(i)}}{d_{\max }}
$$

$$
E i=\frac{E 0_{N(i)}-E_{N(i)}}{E 0_{N(i)}}
$$

Cluster head will calculate the composite values of the nodes, which are used for selecting the cluster head. c1 and c2 are proper impact factors determined by different application scenarios. Equation (2) is the ratio of consumed energy $E_{N(i)}$ to the initial energy $E 0_{N(i)}$ of node i. Equation (3) is the ratio of the distance $d_{N(\mathrm{i})}$ from node $\mathrm{i}$ to the center of mass to the maximum distance $d_{\max }$ from a node in this cluster to the center of mass. The node with minimum $D i$ is chosen to be the next cluster head. Upon completion of the cluster head selection calculation, the cluster head will broadcast a message. Members in the same area as this cluster head changes the cluster head. Node not in the same area as this cluster head ignores this message, and next round begins.

(5) After the completion of the data transmission, if $r \bmod (\mathrm{N}+1)==0$, all members send the information of their surplus energies and locations to their respective cluster head. The cluster heads send the information of all members' surplus energies and locations as well as their owns to the next cluster head or directly to the base station. Then next round begins.

LECCBRA ensures an more uniform distribution of cluster heads and gives rise to an optimal number of cluster heads. At the same time, considering the variation of energy consumptions and nodes fade of each cluster, LECCBRA clusters the network again every other $\mathrm{N}$ rounds, which can balance the energy consumption of the whole network. And in each of $\mathrm{N}$ rounds, the surplus energy and position are both considered for the selection of cluster head, which not only reduces the chance of early vanishing of selected cluster head, but also reduces the energy consumption of member nodes. In this way, the whole energy consumption of the cluster is reduced.

d) Inter-cluster routing: Inter-cluster routing protocol algorithm of LECCBRA is as follows:

(1) If $r \bmod (N+1) !=1$, each cluster head sends the information of remaining energy and position directly to the base station in the beginning.

(2) The base station calculates the distances between any two cluster heads. Then a directed weighted graph $\mathrm{G}$ of all cluster heads is formed. The energy consumption of inter-cluster communication applies to free space channel model, which means the energy consumption increases with the square of the distance. Hence, the weight of two cluster heads is equal to the square of the distance. Then the shortest routing can 
be found between the base station and the rest of cluster heads, using a global shortest path algorithm named Floyd-warshall.

(3) Let $n$ represent the number of cluster head and $V$ be the set of cluster head. The initial value of $V$ is the set of all the cluster heads. The $\operatorname{arcs}[i][j]$ denotes the value between $v_{i}$ and $v_{j}(i, j=1,2,3, \cdots, n)$. We set the CHPARENT_ID of all cluster heads as the ID of the base station. D means the set of the shortest path between the base station (bs) and other cluster heads, and the initial value of $\mathrm{D}[\mathrm{i}]=\operatorname{arcs}[\mathrm{bs}][\mathrm{i}], \mathrm{v}_{\mathrm{i}} \in \mathrm{V}$.

(4) Choose $v_{j}$ and let $D[j]=$ Min $\left\{D[i] \mid v_{i} \in V-S\right\}$. Thus, $v_{j}$ is the end point of the shortest path that starts from the base station. Set $\mathrm{S}=\mathrm{S} \cup\{\mathrm{j}\}$. Choose $\mathrm{v}_{\mathrm{k}}$ that belongs to $\mathrm{V}-\mathrm{S}$, and change the value of the shortest path length from $v_{k}$ to the base station. If $D[j]+\operatorname{arcs}[j][k]<D[k]$, let $D[k]=D[j]+\operatorname{arcs}[j][k]$, and set CHPARENT_ID of cluster head $\mathrm{k}$ as the ID of cluster head $\mathrm{j}$.

(5) Repeat (4) n-1 times.

(6) For all cluster heads, do the following below. Save the CHCHILD_LIST according to CHPARENT_ID. Then broadcast the information of each cluster head. When cluster heads receive a message, they will save their own CHHEAD_ID, CHPARENT_ID and CHCHILD_LIST. The parent node makes a schedule for child nodes, and the child node sends the data collection directly to the parent node in the specified period of time. Figure 1 and Figure 2 show the comparisons of the data transmission of LEACH and

\section{LECCBRA respectively}

\section{CBTS Protocol: Description of CBTS}

e) Synchronization between the base station and cluster head node: The synchronization of cluster head nodes can be divided into 2 steps:

(1) The base station sends a message to cluster heads. Then the cluster heads that receive the information adjust their clocks by Pair-Wise Synchronization to achieve the synchronization. Because the nodes in wireless sensor networks hardly move, the transmission time between a cluster head and the base station is approximately constant. After the first synchronization, the cluster head i records the transmission time di between the base station and itself.

\section{Figure 1: Data transmission of LEACH, LEACH-C}

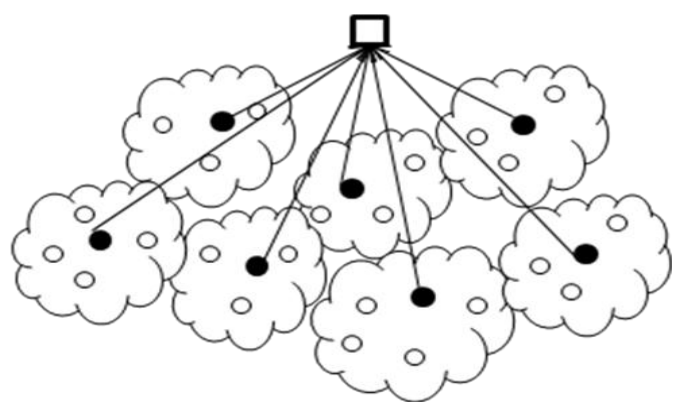

Figure 2: Data transmission of LECCBRA

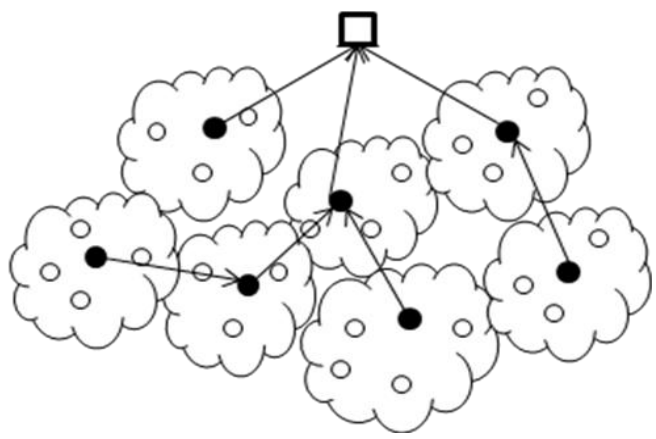


(2) When another round begins, the base station sends a broadcast packet bsy_mesg at $t 1$. The cluster head $\mathrm{i}$ receives the packet at ti. According to $\mathrm{ti}=\mathrm{t} 1+\mathrm{di}+\mathrm{Di}$, calculate the time difference $\mathrm{Di}$, then the synchronization can be achieved. In this way, the base station simply transfer one data packet, thus transmission energy is saved. LECCBRA will transform the cluster head after each data fusion, so it should run the first step after each data fusion, then regularly run the second step. When there is no data fusion, it only needs to run step 2 .

f) Inter-cluster synchronization: When synchronization between the base station and the cluster heads comes to end, the inter-cluster synchronization begins. Synchronization between a cluster head and cluter members can be divided into 2 steps:

(1) The cluster head c broadcasts the synchronization information sy_bg. According to pair-wise synchronization, synchronization between the cluster head $\mathrm{c}$ and node $\mathrm{j}$ is achieved. The cluster head $\mathrm{c}$ saves the time difference $\mathrm{Dj}$ and transmission time dj.

(2) The cluster head c broadcasts a synchronization information csy_mesg to other cluster members, which records the transmission time dj and the time d1 that cluster head was sending csy_mesg. The transmission time between the cluster head and cluster members can be seen the same, which is recorded as d. So when another cluster node k receives csy_mesg, it records the reception time tk and will get the time difference $\mathrm{Dk}=\mathrm{tk}-\mathrm{d}-\mathrm{t} 1$. This way can significantly reduce the energy consumption of synchronization. LECCBRA will change the cluster head with each round of data fusion, but only transform the cluster structure after $\mathrm{N}$ rounds. So it is not necessary to run two steps each round, only step 2 needs to be run regularly.

Performance evaluation of CBTS: This section will evaluate the performance of CBTS through error analysis, energy consumption analysis.

g) Error analysis: We examine the synchronization error by analyzing the sources of error. Figure 3 shows the timeline of CBTS.

\section{Figure 3: The synchronization error of CBTS}

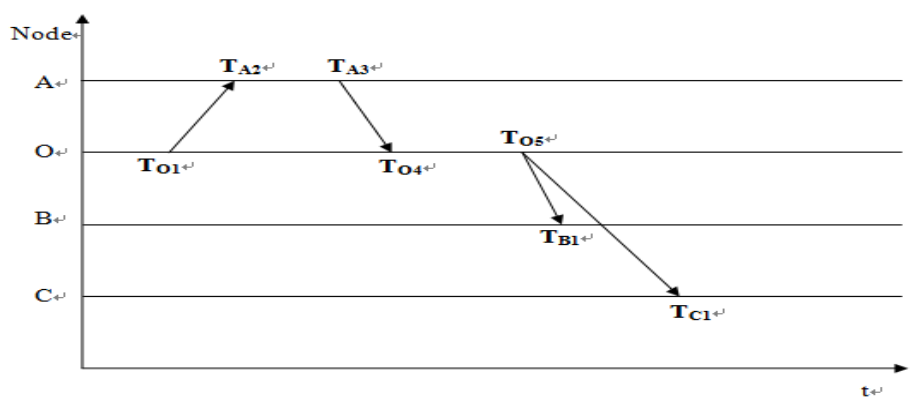

The synchronization of CBTS can be divided into two kinds. The synchronization between the base station and cluster heads is pair-wise synchronization, and the synchronization between cluster head and cluster members is one-way broadcast synchronization. Therefore, the synchronization error of CBTS also can be divided into two kinds. The error between node $\mathrm{A}$ and node $\mathrm{O}$ is pair-wise synchronization error, which is shown as follows:

Error $=\square_{A, o-D_{t_{o 4}}^{O \rightarrow A}}=\frac{S}{2}+\frac{P}{2}+\frac{R}{2}+\frac{R D_{t_{o \rightarrow} \rightarrow t_{04}}^{O \rightarrow A}}{2}$

The synchronization error between node $\mathrm{B}(\mathrm{C})$ and node $\mathrm{O}$ are one-way broadcast synchronization (Fig. 3), which is shown as follows:

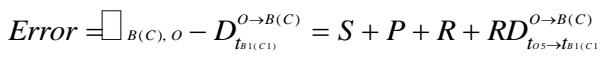

h) Energy consumption analysis: CBTS can effectively reduce energy consumption. It is achieved by reducing the number of data packets. The entire synchronization process only needs to transmit six 
packets, including three packets for the synchronization of the cluster head and three packets for the synchronization of the cluster head members. And in most of the period, it only needs to transmit two packets. In some classical algorithms, such as RBS, TPSN, if the network consists of m nodes, it has to transfer $2 \times(m-1)$ packets to synchronize.

Simulation Results and Analysis of LECCBRA Protocol and CBTS Protocol: In this paper, we use an open source simulation tool: NS2 (Huanget al., 2007) to simulate LECCBRA protocol and CBTS protocol. The simulation is set up as follows. 100 sensor nodes are randomly deployed in the 100 meters times 100 meters area. The coordinates of the base station is at $(100,100)$, and the initial energy for each node is $1 \mathrm{~J}$ (Joule). The consumption energy of sending and receiving data is $50 \mathrm{~nJ}$ per bit, and the consumption of data fusion is $5 \mathrm{~nJ}$ per bit. The size of the packets is 2,000 bit. Specifically, we compare the LECCBRA protocol with LEACH-C protocol and LEACH protocol in three aspects: (1) the number of surviving nodes; (2) the total energy consumption; and (3) the total energy consumption of each round.

\section{Comparison of the number of surviving nodes in each round}

Figure 4 gives a comparison of the number of surviving nodes. For LEACH and LEACH-C protocol, nodes begin to die at about 100-th round, while for LECCBRA, this happens at about 160-th round. Nodes are all dead at rounds of 326 and 374 for LEACH and LEACH-C protocols, while for LECCBRA protocol, the all nodes are still surviving at round of 472. For LECCBRA, the number of surviving nodes in each round is greater than or equal to that for LEACH and LEACH-C. This shows that LECCBRA effectively balances the energy consumption of the whole network, and avoids the situation of some nodes being dead prematurely due to overload. When LECCBRA uses CBTS to achieve time synchronization, the all nodes are still surviving at round of 503. This shows CBTS is suitable for LECCBRA. Integration of LECCBRA with CBTS significantly extends the network life cycle.

\section{Figure 4: Comparison of the number of surviving nodes}

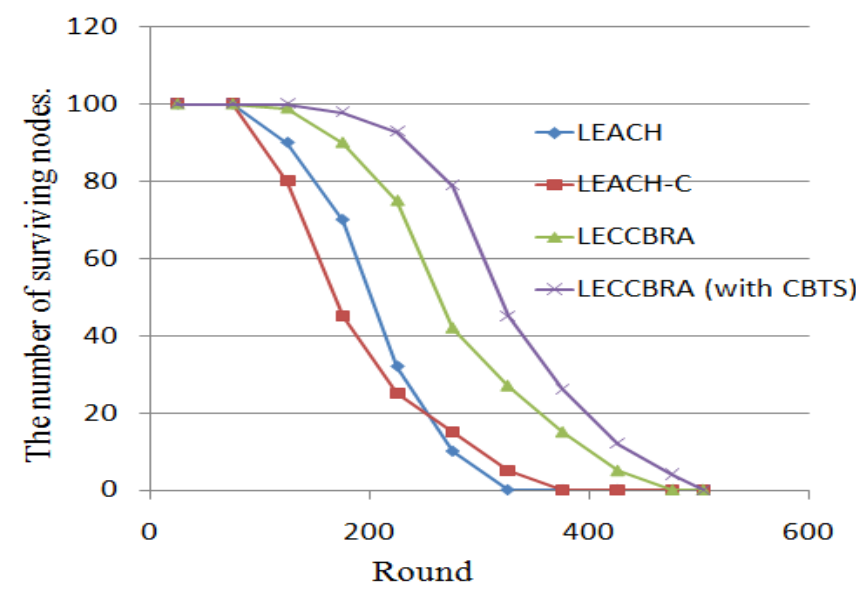

Comparison of total energy consumption: Figure 5 shows a comparison of total energy consumption. As shown in the figure, the energy consumption of LEACH-C is slightly less than that of LEACH. This is because LEACH-C always establishes the optimal cluster structure. Though the establishment of the optimal cluster structure requires additional overhead, once the optimal structure of the clusters is formed, it will effectively reduce the energy consumption of each cluster. But when the number of nodes is small, this becomes less significant. The total energy consumption of LECCBRA is always lower than that of LEACH and LEACH-C protocols. This can be explained by the fact that LECCBRA has not only taken the advantages of the cluster head selection algorithm of LEACH-C to form the optimal cluster structure, but also refined the process of inter-cluster routing. It can be seen from the figure that LECCBRA with CBTS saves more energy at the beginning. This is because the number of transmission packages of normal time synchronization algorithm, like TPSN, is decided by the node number. LECCBRA with CBTS effectively 
reduces the cost of communication between each cluster head and the base stations, thus the energy can be saved.

Figure 5: Comparison of total energy consumption

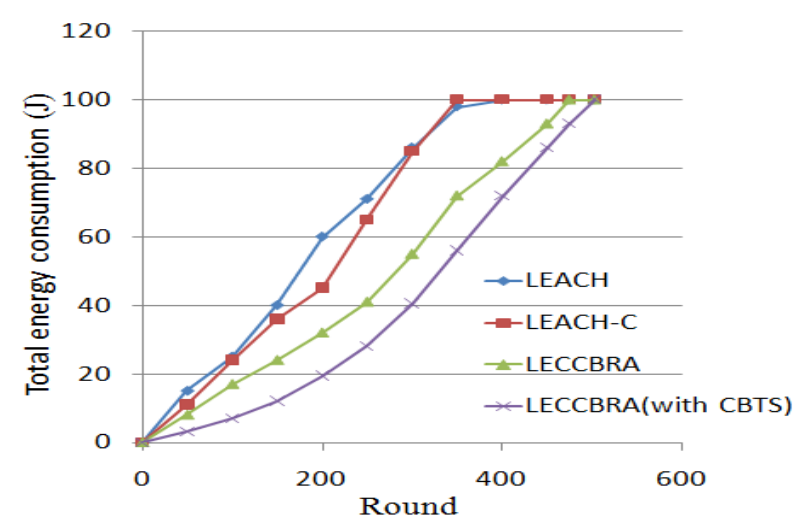

Comparison of total energy consumption per round: Figure 6 shows the comparison of total energy consumption of each round. As one can see from it, for the LEACH protocol, energy consumption fluctuates greatly with each round, while the fluctuations for LEACH-C and LECCBRA are relatively small. This is mainly because LEACH randomly selects the cluster heads, and does not control the number and location of cluster heads, which leads to a bad solution of clustering. Meanwhile, based on the global cluster information, LEACH-C generates the optimal structure in every round. The best cluster structure can reduce the energy consumption of the whole network; so, in most of rounds LEACH-C consumes less energy than LEACH. In addition, LECCBRA uses the cluster head selection algorithm of LEACH-C to uniformly partition the network, thus, the energy consumption of each round is also very uniform. It also uses multi-hop approach to complete the communication between cluster heads and base stations, and in the cluster it decides the next cluster head by considering the remaining energy and location of cluster members. Therefore, LECCBRA consumes less energy than LEACH and LEACH-C per round. LECCBRA with CBTS saves more energy. This is because CBTS effectively reduces the number of transmission packages.

Time accuracy of CBTS: Use NS2 to simulate CBTS. Verify the advantages of CBTS algorithm by analyzing the relationship between the time accuracy and the number of hops. The payload of transmitting a packet is 38 bytes. The communication bandwidth is $250 \mathrm{kbps}$. The MAC layer uses the 802.11 . The delay time model of the sender and receiver is a Gaussian distribution model. Figure 7 shows that node's time synchronization accuracy increases when the number of hops increases. CBTS only has two-hop, so the accuracy is significantly improved.

\section{Figure 6: Comparison of the total energy consumed per round}

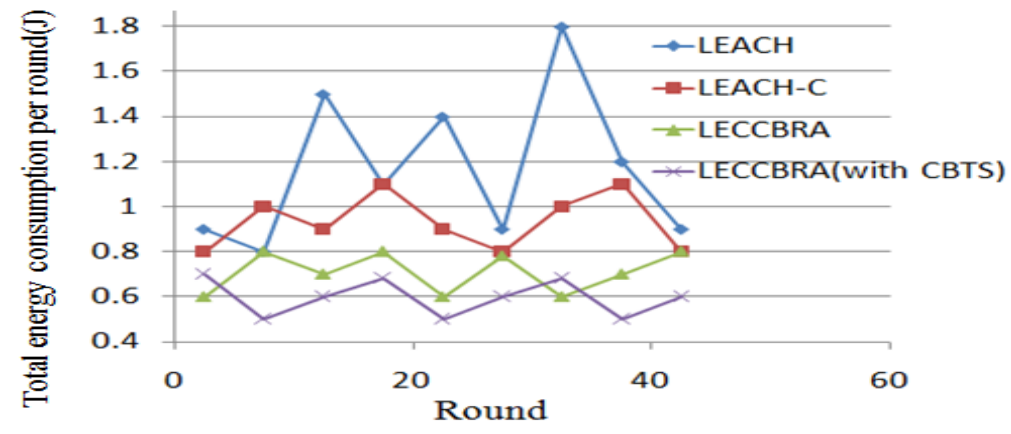


Figure 7: Relationship between time accuracy and the number of hops

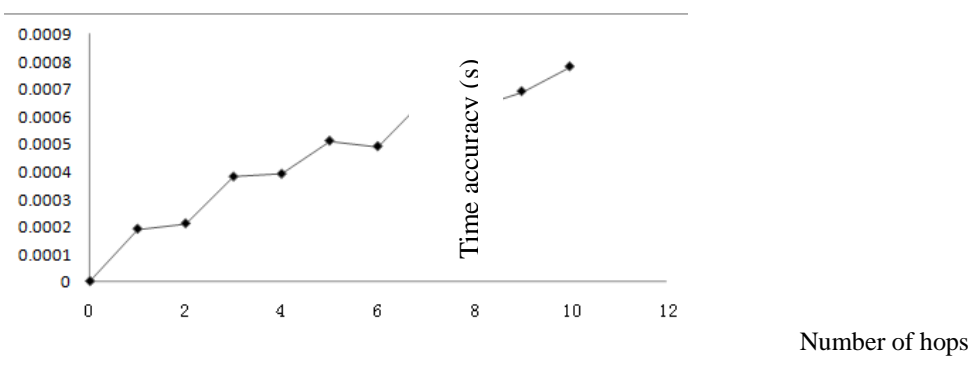

\section{Conclusion}

LECCBRA algorithm and CBTS algorithm are proposed in this paper. LECCBRA regularly uses the LEACH-C cluster head selection algorithm to partition the network for controlling the number and location of cluster head. As a result, the cluster heads are equally distributed in the network area, and the number of cluster heads is neither too big nor too small. For the rest of rounds not using LEACH-C algorithm, the one with more remaining energy and being closer to the center of mass is selected to be the next cluster head. In this way, the consumption of the inter-cluster can be balanced, and the nodes do not need to report to the base station in each round, which will reduce energy cost greatly. LECCBRA uses the inter-cluster multi-hop mode to complete the communications between cluster heads and the base station. The energy factor is considered in choosing the cluster head that directly communicates with the base station, and the global shortest path algorithm is used to minimize the total routing consumption. Simulation results show that LECCBRA outperforms LEACH and LEACH-C protocols in terms of the number of surviving nodes and the energy consumption. To conclude, LECCBRA can effectively save energy, balance energy consumption and prolong the network lifespan. CBTS establishes the synchronization between the base station and the cluster heads at first, and then does the synchronization of cluster members. The performance analysis and simulation results show that the proposed time synchronization algorithm not only saves the energy of the system by reducing the transmission of data packets, but also improves the accuracy. In addition, CBTS is very suitable for LECCBRA and applications in wireless sensor networks.

\section{References}

Commission of the European Communities. (2009). Internet of Things-An action plan for Europe, 1st ed.. Brussels: COM, 278, 1-12.

Floyd, R. W. (1962). Algorithm 97: Shortest path. Commun. ACM, 5(6), 345.

Heinzelman, W., Chandrakasan, A. \& Balakrishnan, H. (2000). Energy - efficient communication protocols for wireless micro sensor networks, In: Procs. of 33rd Hawaii Intl Conf on Systems Sciences, 3005 $-3014$.

Huang, H. J., Feng, S. L. \& Qin, L. J. (2010). NS network simulation and protocol emulation. Beijing: Posts \& Telecom Press.

Kranenburg, R. V. (2007). The Internet of Things: A critique of ambient technology and the all-seeing network of RFID, in Network Notebooks 02. Amsterdam: Institute of Network Cultures Press, 10-28.

Ren, F. Y., Huang, I. I. \& Lin, C. (2003). Wireless sensor networks. Journal of Software, 14(2), 1148-1157.

Siva, D. M. G. \& Ma, D. C. F. (2005). A Centralized energy-efficient routing protocol for wireless sensor networks. IEEE Radio Communications, 43(3), 8-13. 